\title{
Id proteins expression in prostate cancer: high-level expression of Id-4 in primary prostate cancer is associated with development of metastases
}

\author{
Hiu-Fung Yuen ${ }^{1}$, Chee-Wai Chua ${ }^{2}$, Yuen-Piu Chan ${ }^{1}$, Yong-Chuan Wong ${ }^{2}$, Xianghong Wang ${ }^{2}$ \\ and Kwok-Wah Chan ${ }^{1}$ \\ ${ }^{1}$ Department of Pathology, Faculty of Medicine, The University of Hong Kong, Pokfulam, Hong Kong, \\ China and ${ }^{2}$ Department of Anatomy, Faculty of Medicine, The University of Hong Kong, Pokfulam, \\ Hong Kong, China
}

\begin{abstract}
A major cause of treatment failure for prostate cancer is the development of androgen-independent metastatic disease. Id protein family, a group of basic helix-loop-helix transcription factors, has been shown to be involved in carcinogenesis and a prognostic marker in several types of human cancers. In this study, we examined the expressions of four Id proteins, Id-1, -2, -3 and -4, in 125 clinical prostate cancer specimens as well as 40 nodular hyperplasia specimens by immunohistochemistry. The expressions of Id proteins were correlated with Gleason grading and metastatic progress of the tumors. We found that Id proteins were dysregulated in prostate cancer. Id-1 and -2 expressions were elevated while Id-3 and -4 expressions were reduced in prostate cancers compared to nodular hyperplasia. Cytoplasmic staining of $I d-1(P=0.013)$ and nuclear staining of Id-2 $(P=0.001)$ and Id-4 $(P<0.001)$ were positively correlated with Gleason score. The results indicate that these Id proteins may play a positive role in the development of prostate cancer. In contrast, Id-3 might have an inverse relationship with prostate neoplastic transformation $(\boldsymbol{P}=0.002)$ and cancer progression $(P=0.022)$. We found that Id-4 nuclear overexpression in the primary prostate cancers significantly increased the risks to the development of metastasis in the patients (odds ratio $=3.215,95 \%$ confidence interval $=1.150-$ $8.987, P=0.026$ ). Our results suggest that in prostate cancer patients, differential Id proteins expressions may be a useful marker for poor prognosis, and Id-4 may be a potential prognostic marker for distant metastasis. Modern Pathology (2006) 19, 931-941. doi:10.1038/modpathol.3800602; published online 31 March 2006
\end{abstract}

Keywords: Id; prostate cancer; metastasis

In Asian populations, there is a trend of increasing incidence of prostate cancer which may be due to the adoption of Western lifestyle. ${ }^{1}$ Owing to the relatively slow growth rate of many prostate cancers, newly diagnosed cases, especially in elderly men, may be of little clinical significance. Treatment may not be of benefit to these patients and watchful waiting is an acceptable form of patient management. ${ }^{2,3}$ On the other hand, approximately one-third of all prostate cancer patients have micro-metastatic

Correspondence: Dr X Wang, PhD, Department of Anatomy, The University of Hong Kong, 1/F, Faculty of Medicine Building, 21 Sassoon Road, Hong Kong.

E-mail: xhwang@hkucc.hku.hk

Dr KW Chan, MBBS, FRCPath, Department of Pathology, The University of Hong Kong, Hong Kong.

E-mail: kwchan@pathology.hku.hk

Received 12 January 2006; revised 8 March 2006; accepted 9 March 2006; published online 31 March 2006 disease at the time of presentation. This group of patients is largely undetected due to a lack of reliable biomarkers. These patients eventually progress to clinically detectable metastatic and androgen-independent diseases at which point the median survival is only 12-15 months. ${ }^{4}$ Therefore, prognostic markers that identify prostate cancer patients at risks of developing metastatic lesion would be able to facilitate proper patient care.

Inhibitors of Differentiation/Inhibitors of DNA bin̄ing (Id) protein family, a group of basic helixloop-helix transcription factors, is involved in negative regulation of other bHLH transcription factors in many cellular processes as well as in carcinogenesis. Its involvement in cell cycle control,$^{5}$ cancer development, ${ }^{6}$ angiogenesis ${ }^{7}$ and apoptosis $^{8}$ has been implicated. Therefore, it is regarded to be a potential target for cancer therapy. ${ }^{9}$ There are four members in this protein family, namely Id-1, 
Id-2, Id-3 and Id-4. ${ }^{10}$ Although they are in the same family, their roles in carcinogenesis in different types of cancer are different.

Id-1, -2 and -3 have been shown to participate in early stages of hepatocarcinogenesis but not in liver cancer progression, while reduced expressions of Id-1 are associated with a better prognosis in hepatocellular carcinoma. ${ }^{11}$ Id-1 has been suggested to be a prognostic marker in cervical cancer, in which higher expressions of Id-1 were correlated with a poor prognosis. ${ }^{12}$ Colorectal adenocarcinoma tumor specimens had elevated expressions of Id-1, -2 and -3 when compared to normal tissue, ${ }^{13}$ while hypermethylation with reduced expressions of Id-4 correlated with a poor prognosis. ${ }^{14}$ Id-1 and -2 were overexpressed in pancreatic cancer and could be early markers of pancreatic malignant transformation. ${ }^{15}$ Overexpression of Id-1 has also been described in gastric cancer, in which Id-1 expression was positively correlated with tumor progression. ${ }^{16}$ Id-4, on the other hand, was hypermethylated and reduced in this cancer. ${ }^{17}$ In breast cancer, high cytoplasmic expressions of Id-2 were found to confer a favorable prognosis, ${ }^{18}$ while high nuclear expressions of Id-4 were shown to correlate with cancer progression. Id-3 expression was reduced in ovarian cancer. ${ }^{19}$ These results suggest that the expression of different Id proteins in different types of cancer could be used as prognostic markers of cancers.

The roles of Id proteins in cancer metastasis have also been studied. The metastatic potential of gastric cancer cells was significantly reduced in Id1 and -3 double-knockdown cells. ${ }^{20}$ Id-1 was highly expressed in infiltrating human breast cancer specimens,${ }^{21}$ while Id-2 expression was barely detectable in invasive human breast cancer specimens. ${ }^{22}$ Id-4 expressions were shown to correlate with invasiveness of breast cancer cells ${ }^{23}$ and breast carcinomas in mice. ${ }^{24}$ However, another study has suggested that hypermethylation of Id-4, which leads to reduced gene expression, is significantly associated with node-positive T1 primary breast cancer. ${ }^{25}$ These findings show that Id proteins may also be involved in metastasis of different types of cancer.

Our previous study has shown that Id-1 is overexpressed in human prostate cancer $(n=47)$, and the poorly differentiated prostate cancer shows stronger expressions. ${ }^{26}$ Our results were confirmed by others when they demonstrated that Id-1 was upregulated during human prostate cancer progression and that prostate cancer cells showed increased proliferation and invasiveness when they overexpressed Id-1. ${ }^{27}$ In the same study, Id-2 was shown to be upregulated in prostate cancer. Id-2 expression promoted prostate cancer cells to a more aggressive phenotype, though it was less potent than Id-1.

The aims of our present study were to study how the Id proteins expressions were related to prostate carcinogenesis, cancer progression and development of metastasis in human prostate cancer. We also explored whether differential expressions of Id proteins might be prognostic markers of prostate cancer progression and metastasis. To investigate expressions of the Id proteins in prostate cancer, we studied 125 prostate cancer specimens by immunohistochemical staining in tissue microarray and had them compared to 40 nodular hyperplasia samples. We found that there was significant difference in the expressions of Id proteins between prostate cancer and nodular hyperplasia. Upregulation of cytoplasmic Id-1, nuclear Id-2 and nuclear Id-4 expressions were positively associated with Gleason score while Id-3 expression was negatively associated. In addition, primary prostate cancer specimens with high nuclear Id-4 expressions were at a higher risk of developing metastatic disease. Our results suggest that Id proteins are involved in neoplastic transformation and progression of prostate cancer, and Id-4 may be a useful marker for the prediction of distant metastasis.

\section{Materials and methods}

\section{Patients and Specimens}

A total of 165 archival formalin-fixed and paraffinembedded prostate specimens were obtained from Department of Pathology, The University of Hong Kong at Queen Mary Hospital. The specimens included 40 cases of nodular hyperplasia, and 125 cases of prostate cancer, which were derived from 122 cases of transurethral resections, 16 cases of radical prostatectomies, and 27 cases of needle biopsies. The median age of the patients at the time of procurement of the tissue samples is 76 (range 55-94).

The archival dates of the prostate cancer specimen fell between years 1998 and 2004. The prostate specimens were divided into the Benign $(n=40)$ and Cancer $(n=125)$ groups. The prostate cancer specimens were reviewed and graded histologically according to Gleason's method. They were divided into two groups, one with a combined Gleason score less than $7(n=38)$ and Gleason score equal to or more than $7(n=87)$. In order to correlate between Id proteins expressions and development of metastatic diseases in Gleason score matched specimens, the Gleason score $\geq 7$ group of patients were then subdivided according to their metastatic status, nonmetastatic (no metastases detected (median follow-up 38 months, ranged from 12 to 90 months) after diagnosis, $n=34$ ) and metastatic (distant metastases detected within 6 months after procurement of the specimens, $n=37$ ) groups. All prostate cancer patients selected for this study did not receive any therapy directed against prostate cancer prior to procurement of the specimens. Prostatespecific antigen level at the time of specimen procurement was available for 92 prostate cancer 
Table 1 Patient clinical and pathologic features

\begin{tabular}{|c|c|c|c|}
\hline & $\begin{array}{l}\text { Number } \\
\text { of cases }\end{array}$ & $\%$ & Median (range) \\
\hline Histological group & 165 & & \\
\hline Nodular hyperplasia & 40 & 24 & \\
\hline $\begin{array}{l}\text { Prostate cancer } \\
(\text { Gleason score }<7 \text { ) }\end{array}$ & 38 & 23 & \\
\hline $\begin{array}{l}\text { Prostate cancer } \\
(\text { Gleason score } \geq 7)\end{array}$ & 87 & 53 & \\
\hline Age & & & $76(55-94)$ \\
\hline Nodular hyperplasia & 40 & & $76(70-78)$ \\
\hline $\begin{array}{l}\text { Prostate cancer } \\
\text { (Gleason score <7) }\end{array}$ & 38 & & $72.5(56-92)$ \\
\hline $\begin{array}{l}\text { Prostate cancer } \\
\text { (Gleason score } \geq 7 \text { ) }\end{array}$ & 87 & & $74(55-94)$ \\
\hline Nonmetastatic & 34 & & $77(59-94)$ \\
\hline Metastatic & 37 & & $72(56-91)$ \\
\hline Pre-PSA level & 92 & 74 & $\begin{array}{c}62.50 \\
(0.4-3744.0) \mathrm{ng} / \mathrm{ml}\end{array}$ \\
\hline $\mathrm{PSA} \leq 4 \mathrm{ng} / \mathrm{ml}$ & 3 & 3 & \\
\hline $4 \mathrm{ng} / \mathrm{ml}<\mathrm{PSA}<10 \mathrm{ng} / \mathrm{ml}$ & 15 & 16 & \\
\hline $\mathrm{PSA} \geq 10 \mathrm{ng} / \mathrm{ml}$ & 74 & 80 & \\
\hline Metastatic status $(G S \geq 7)$ & 71 & & \\
\hline Nonmetastatic & 34 & 47 & \\
\hline $\begin{array}{l}\text { Metastatic } \\
\text { (within } 6 \text { months) }\end{array}$ & 37 & 52 & \\
\hline Sites of metastases detected & 37 & & \\
\hline Bone & 35 & 95 & \\
\hline Lymph node & 2 & 5 & \\
\hline
\end{tabular}

pre-PSA, preoperative prostate-specific antigen.

cases $(74 \%)$. The clinical information of the patients is summarized in Table 1.

\section{Tissue Microarray Construction}

Tissue microarray was constructed with a Beecher Instruments tissue microarrayer (Silver Spring, MD, USA). A pathologist (Chan KW) marked three representative areas for each specimen other than a needle biopsy. The selected areas were incorporated into a paraffin block using a 0.6-mm diameter core needle. A total of six tissue microarray blocks were made each containing $\sim 80$ cores. H\&E stained sections of the tissue microarray blocks were reviewed by Chan KW to ensure that targeted tissue had been sampled before performing further studies. The sample numbers varied slightly among staining results of the four Id proteins because of variability in the number of interpretable specimens on tissue microarray sections.

\section{Immunohistochemical Staining}

Immunohistochemical staining was carried out as described previously ${ }^{26}$ using ABC Vectastain Kit (vector Laboratories, Burlingame, CA, USA), according to the manufacturer's instructions.

\section{Evaluation of Immunohistochemical Staining Results}

The stained sections were reviewed by two independent observers (Chan KW and Chan YP), who had no knowledge of the patient data. All three cores of each sample were examined and the core with the highest staining intensity and extent was selected for further quantification. In case of a needle biopsy, the whole section was evaluated and the 'hot spot' showing the highest staining intensity was selected for further quantification. Cytoplasmic staining was scored by the extent and intensity of staining, which was then graded by an arbitrary scale that ranged from 0 to 3, representing negative ('0'), weak (' 1 '), moderate ('2') and strong ('3') staining intensity, respectively. Nuclear staining was scored by the percentage of nuclei positively stained in the hot core/hot area observed under $\times 400$ magnification with at least 100 nuclei included, and the result was expressed as mean \pm s.e.m.

\section{Statistical Analysis}

Statistical analysis was performed using SPSS 13.0 software. Differences in Id proteins expressions among different groups of specimens were analyzed by independent samples $t$-test and nonparametric Mann-Whitney U-test where applicable. The association between cytoplasmic or nuclear staining of Id proteins and the risk to develop metastatic disease was estimated by odds ratio and 95\% confidence intervals using binary logistic regression analysis. For cytoplasmic staining, negative and weak staining results were classified as low-level expression, and moderate and strong staining results classified as high-level expression. For nuclear staining, the median percentage of nuclei stained within Gleason score matched group for metastasis analysis was used as the cutoff point. The cutoff points for Id-2 and -4 are 1.47 and $32.4 \%$, respectively. Spearman rank correlation test was applied to analyze the correlation between individual Id protein expression and Gleason score. A $P$-value $<0.05$ was considered statistically significant.

\section{Results}

\section{Id Proteins were Differentially Expressed in Benign Prostate and Prostate Cancer}

To investigate whether Id proteins are dysregulated in prostate cancer, we analyzed expressions of Id proteins in benign prostate $(n=40)$ and prostate cancer $(n=125)$ specimens. Representative results of the immunohistochemical staining were shown in Figure 1. Cytoplasmic staining results were summarized in Figure 2c and Table 2. Nuclear staining results of Id-2 and -4 are shown in Figure 2a and $\mathrm{b}$, and Table 3. Cytoplasmic staining of Id-1 was significantly increased in prostate cancer $(P<0.001)$ (Figure 2c). This result is similar to that 
of our previous study. ${ }^{26}$ For Id-2, both cytoplasmic (Figure 2c) and nuclear staining (Figure 2a) was significantly increased in prostate cancer specimens $(P<0.001)$, a finding that was reported by others as well. ${ }^{27}$ In addition, we also observed significantly decreased cytoplasmic expression of Id-3 in prostate cancer specimens $(P=0.002)$ (Figure 2c). Cytoplasmic staining of Id-4 was not significantly different
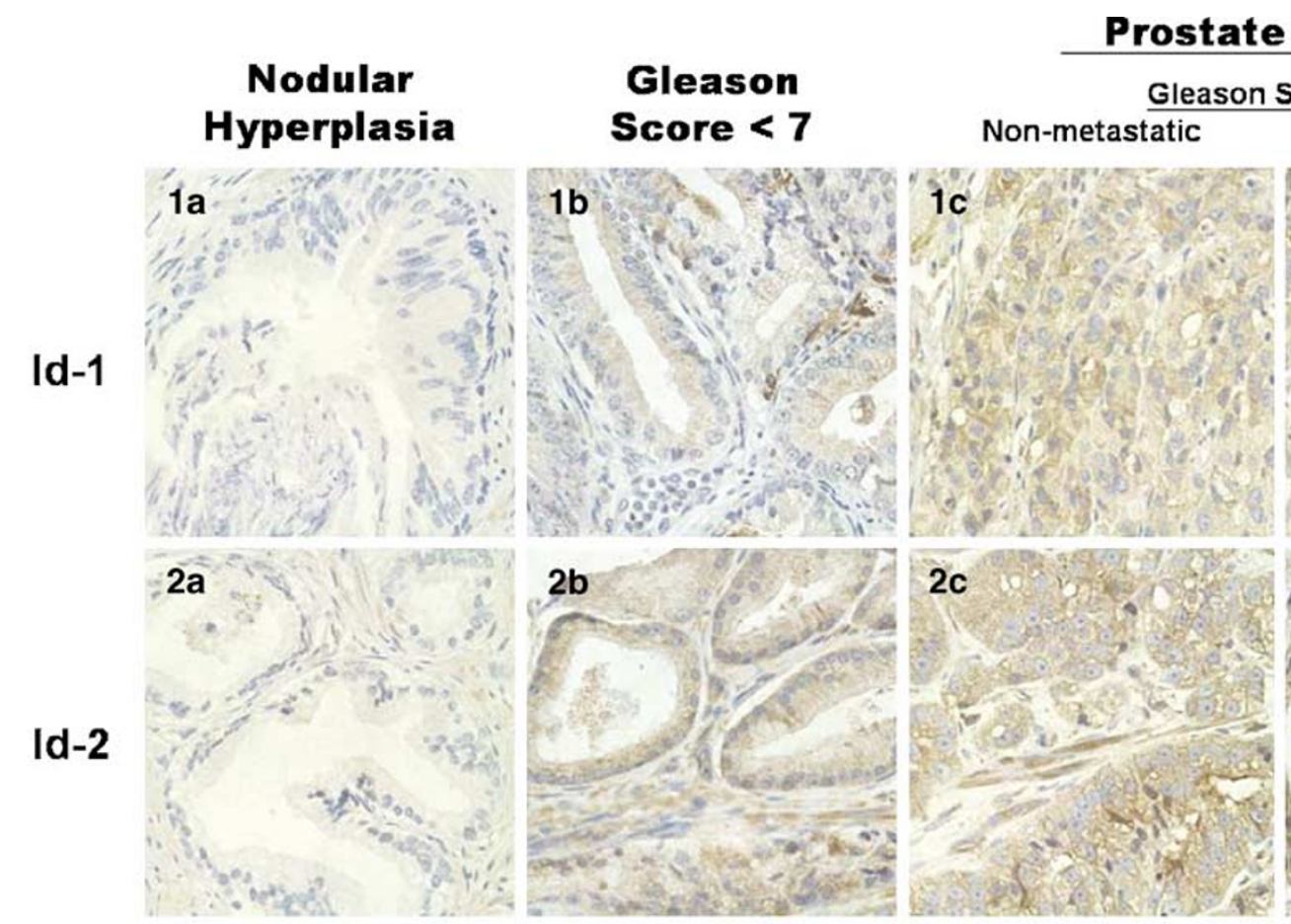

\section{Prostate Cancer}

\section{Metastatic}
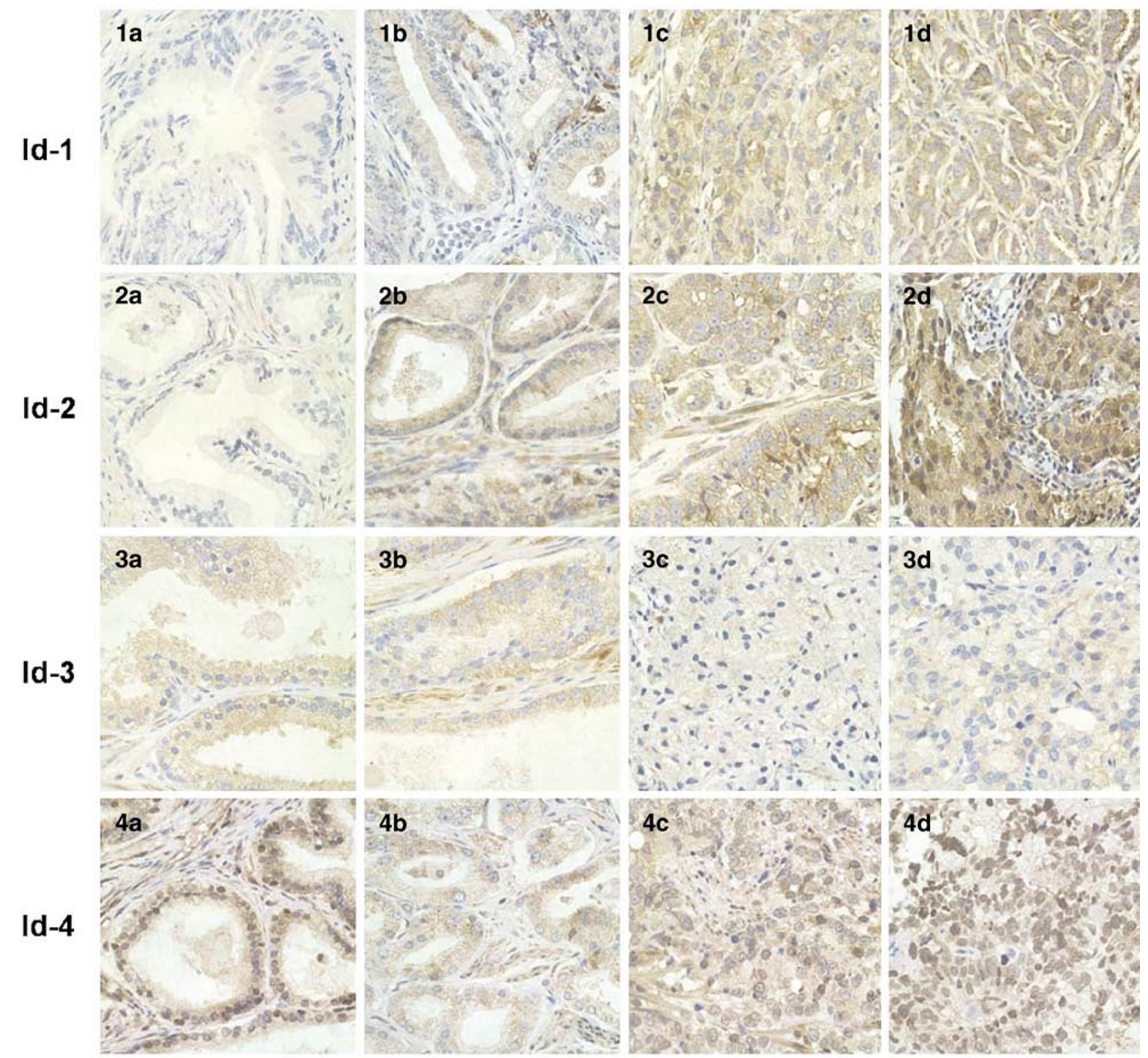

$5 a$

$5 b$

$5 c$

$5 d$ 
$(P=0.541)$ between cancer and benign tissues, but a significant decrease in nuclear Id-4 staining was observed in prostate cancer specimens $(P<0.001)$ (Figure 2b). The results suggest that Id-1 and -2 may play a positive role while Id-3 and -4 may play a negative role in neoplastic transformation of prostate.

\section{Id Proteins were Differentially Expressed during Neoplastic Transformation and Prostate Cancer Progression}

To investigate whether Id proteins are differentially regulated during neoplastic transformation and prostate cancer progression, we analyzed Id proteins
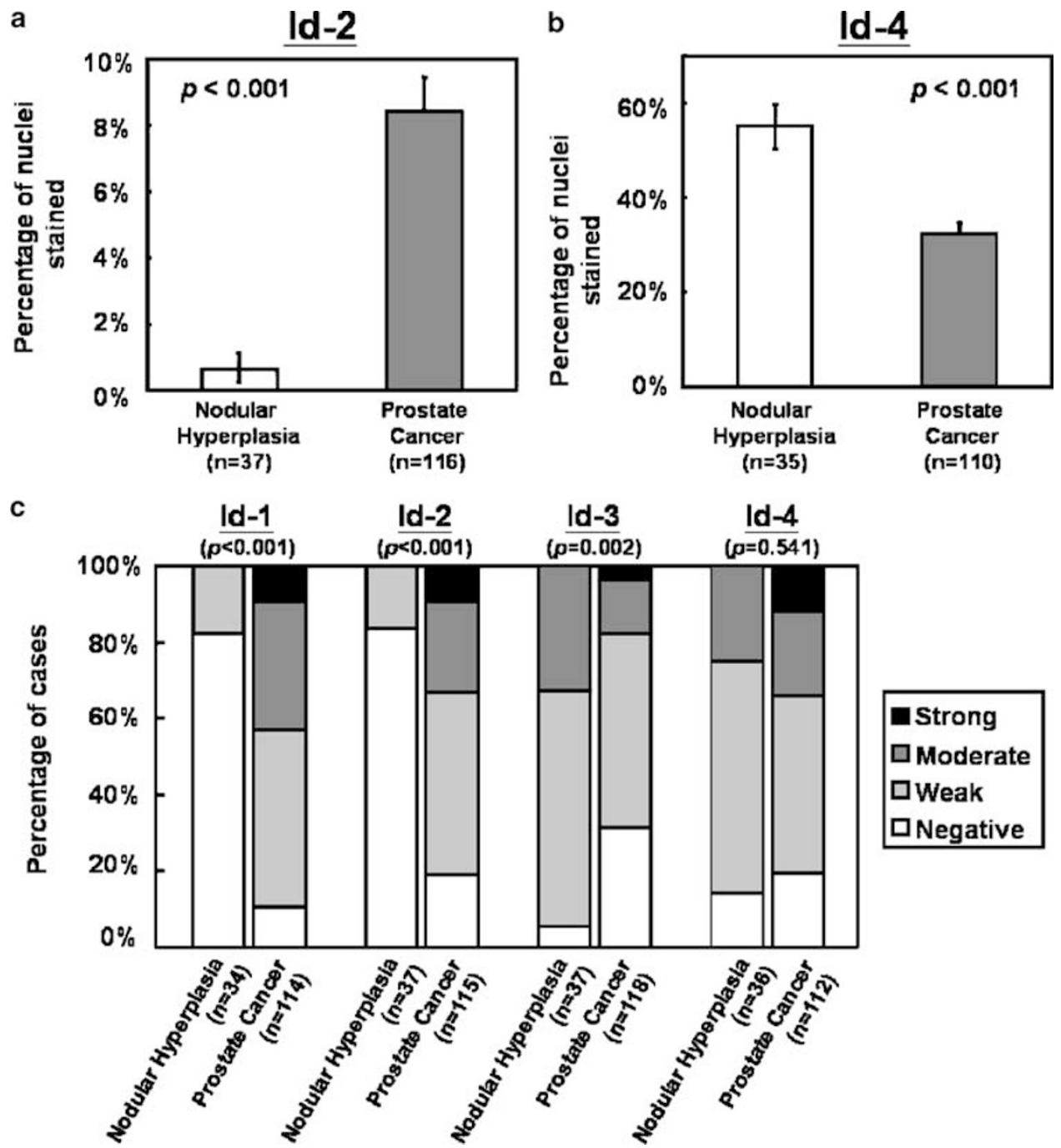

Figure 2 Summary of Id proteins immunohistochemical staining results in nodular hyperplasia and prostate cancer specimens. (a) Percentage of nuclei stained for Id-2. (b) Percentage of nuclei stained for Id-4. (c) Cytoplasmic staining intensity for the four Id proteins. Differences in the level of staining are significant for cytoplasmic Id-1, -2 and -3 expressions and for nuclear Id-2 and -4 expressions. These results suggest Id- 1 and -2 may play a positive role while Id-3 and -4 may play a negative role in neoplastic transformation of prostate.

Figure 1 Immunohistochemical staining of Id proteins in tissue microarray of human prostate specimens. Tissue microarray sections were stained using polyclonal anti-Id-1, $-2,-3$ and -4 antibodies (Santa Cruz Biotechnology, Santa Cruz, CA, USA) in the dilution of 1:500, 1:300, 1:300 and 1:100, respectively. Panels 1, 2, 3 and 4 represent tissue cores stained with Id-1, $-2,-3$ and -4 , respectively at a magnification of $\times 400$. Column A, B, C and D represent nodular hyperplasia, low-grade prostate cancer, high-grade prostate cancer of the nonmetastatic subgroup and high-grade prostate cancer of the metastatic subgroup. Panel 1: Id-1 staining, negative in nodular hyperplasia, increases from low-grade to high-grade cancer. Panel 2: Id-2 shows negative staining in nodular hyperplasia and increased cytoplasmic staining in prostate cancer. Increased nuclear staining in prostate cancer of the metastatic subgroup is observed; Panel 3: Id-3 shows positive staining in nodular hyperplasia with reduced staining in prostate cancer specimens; Panel 4: Id-4 shows positive nuclear staining in nodular hyperplasia and reduced nuclear staining in prostate cancer specimens. However, high level of Id-4 nuclear staining is observed in the primary prostate cancer of the metastatic subgroup. Panel 5: Negative control, primary antibody was omitted during immunohistochemical staining. 
Table 2 Summary of cytoplasmic Id immunohistochemical staining results in different histological stage of prostate specimens

\begin{tabular}{|c|c|c|c|c|c|}
\hline & $\begin{array}{c}\text { Negative } \\
\text { No. of cases (\%) }\end{array}$ & $\begin{array}{c}\text { Weak } \\
\text { No. of cases (\%) }\end{array}$ & $\begin{array}{c}\text { Moderate } \\
\text { No. of cases (\%) }\end{array}$ & $\begin{array}{c}\text { Strong } \\
\text { No. of cases (\%) }\end{array}$ & $\begin{array}{c}\text { Total } \\
\text { No. of cases (\%) }\end{array}$ \\
\hline \multicolumn{6}{|c|}{ (a) Cytoplasmic Id-1 expression } \\
\hline Nodular hyperplasia & $28(82)$ & $6(18)$ & $0(0)$ & $0(0)$ & $34(100)$ \\
\hline \multicolumn{6}{|l|}{ Prostate cancer } \\
\hline Gleason score $<7$ & $5(14)$ & $22(63)$ & $7(20)$ & $1(3)$ & $35(100)$ \\
\hline Gleason score $\geq 7$ & $7(9)$ & $31(39)$ & $31(39)$ & $10(13)$ & $79(100)$ \\
\hline \multicolumn{6}{|c|}{ (b) Cytoplasmic Id-2 expression } \\
\hline Nodular hyperplasia & $31(84)$ & $6(16)$ & $0(0)$ & $0(0)$ & $37(100)$ \\
\hline \multicolumn{6}{|l|}{ Prostate cancer } \\
\hline Gleason score $<7$ & $8(22)$ & $19(53)$ & $9(25)$ & $0(0)$ & $36(100)$ \\
\hline Gleason score $\geq 7$ & $14(18)$ & $36(45)$ & $18(23)$ & $11(14)$ & $79(100)$ \\
\hline \multicolumn{6}{|c|}{ (c) Cytoplasmic Id-3 expression } \\
\hline Nodular hyperplasia & $2(6)$ & $23(62)$ & $12(32)$ & $0(0)$ & $37(100)$ \\
\hline \multicolumn{6}{|l|}{ Prostate cancer } \\
\hline Gleason score $<7$ & $6(16)$ & $22(59)$ & $8(22)$ & $1(3)$ & $37(100)$ \\
\hline Gleason score $\geq 7$ & $31(38)$ & $38(47)$ & $9(11)$ & $3(4)$ & $81(100)$ \\
\hline \multicolumn{6}{|c|}{ (d) Cytoplasmic Id-4 expression } \\
\hline Nodular Hyperplasia & $5(14)$ & $22(61)$ & $9(25)$ & $0(0)$ & $36(100)$ \\
\hline \multicolumn{6}{|l|}{ Prostate cancer } \\
\hline Gleason score $<7$ & $11(32)$ & $15(44)$ & 6 (18) & $2(6)$ & $34(100)$ \\
\hline Gleason score $\geq 7$ & $11(14)$ & $37(47)$ & $19(25)$ & $11(14)$ & $78(100)$ \\
\hline
\end{tabular}

Table 3 Summary of nuclear Id immunohistochemical staining results

\begin{tabular}{|c|c|c|}
\hline & $\begin{array}{l}\text { Number of } \\
\quad \text { cases }\end{array}$ & Mean ( \pm s.e.m.) \\
\hline \multicolumn{3}{|l|}{ Nuclear expression of Id-2 } \\
\hline \multicolumn{3}{|l|}{ Histological group } \\
\hline Nodular hyperplasia & 37 & $0.63(0.44)$ \\
\hline $\begin{array}{l}\text { Prostate cancer } \\
\text { (Gleason score }<7 \text { ) }\end{array}$ & 35 & $5.33(1.75)$ \\
\hline $\begin{array}{l}\text { Prostate cancer } \\
\text { (Gleason score } \geq 7 \text { ) }\end{array}$ & 81 & $9.78(1.23)$ \\
\hline \multicolumn{3}{|l|}{ Metastatic status (GS $\geq 7$ ) } \\
\hline Nonmetastatic & 32 & $5.33(1.29)$ \\
\hline Metastatic (within 6 months) & 34 & $11.72(2.26)$ \\
\hline \multicolumn{3}{|l|}{ Nuclear expression of Id-4 } \\
\hline \multicolumn{3}{|l|}{ Histological group } \\
\hline Nodular hyperplasia & 35 & $55.03(4.62)$ \\
\hline $\begin{array}{l}\text { Prostate cancer } \\
(\text { Gleason score }<7 \text { ) }\end{array}$ & 33 & $22.24(4.10)$ \\
\hline $\begin{array}{l}\text { Prostate Cancer } \\
\text { (Gleason score } \geq 7 \text { ) }\end{array}$ & 77 & $36.65(2.71)$ \\
\hline \multicolumn{3}{|l|}{ Metastatic status (GS $\geq 7$ ) } \\
\hline Nonmetastatic & 29 & $26.22(3.69)$ \\
\hline Metastatic (within 6 months) & 35 & $46.09(4.12)$ \\
\hline
\end{tabular}

staining in nodular hyperplasia $(n=40)$, low-grade (Gleason score $<7, n=38$ ) and high-grade prostate cancers (Gleason score7, $n=87$ ) and correlated the staining results with Gleason score. The findings are summarized in Figure 3, and Tables 2 and 3. Cytoplasmic Id-1 staining was significantly increased in low-grade prostate cancer specimens when compared to nodular hyperplasia $(P<0.001)$, and significantly higher Id-1 expression $(P=0.008)$ was observed in high-grade over low-grade prostate cancer specimens (Figure 3c). A weak correlation of staining results with Gleason score could be established for Id-1 ( $r=0.232, P=0.013)$. Nuclear staining (Figure 3a), but not cytoplasmic staining (Figure 3c), of Id-2 was significantly increased $(P=0.009)$ in high-grade prostate cancer and correlated with Gleason score $(r=0.298, P=0.001)$. Id-3 expression was not significantly different between nodular hyperplasia and low-grade prostate cancer. A significant increase in cytoplasmic staining of Id-3 $(P=0.022)$ was observed in high-grade compared to low-grade prostate cancer (Figure 3c). A weak negative correlation between Id-3 staining and Gleason score was found but was not statistically significant $(r=-0.169, \quad P=0.068)$. Cytoplasmic staining of Id-4 was not significantly different between nodular hyperplasia and low-grade prostate cancer $(P=0.282)$ (Figure 3c), while a significant difference was observed in nuclear Id-4 staining between these two groups $(P<0.001)$ (Figure $3 \mathrm{~b})$. Both cytoplasmic and nuclear staining was significantly increased ( $P=0.025$ and 0.001 , respectively) in high-grade over low-grade prostate cancer, but only nuclear staining was significantly correlated with Gleason score $(r=0.422, P<0.001)$. These results suggest that Id-1 and -2 play a positive role while Id-3 plays a negative role in neoplastic transformation and cancer progression of prostate. In addition, they suggest that Id-4 plays a negative role in neoplastic transformation but a positive role in cancer progression of prostate. 
a

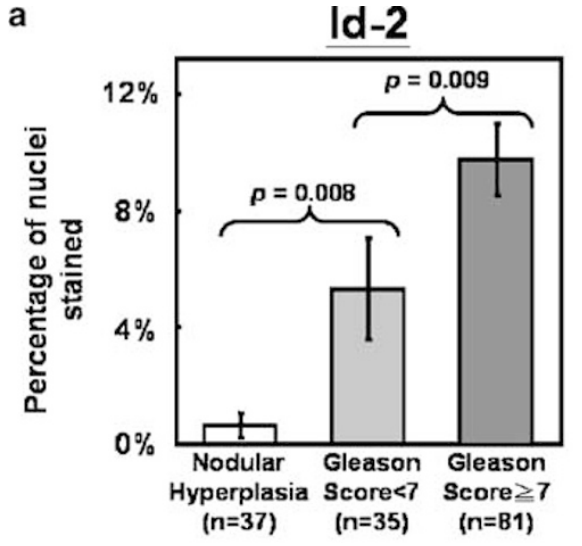

c

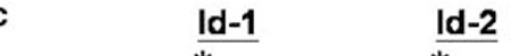

b

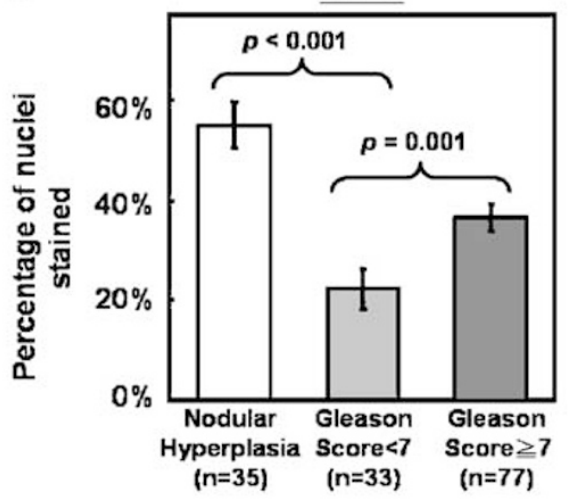

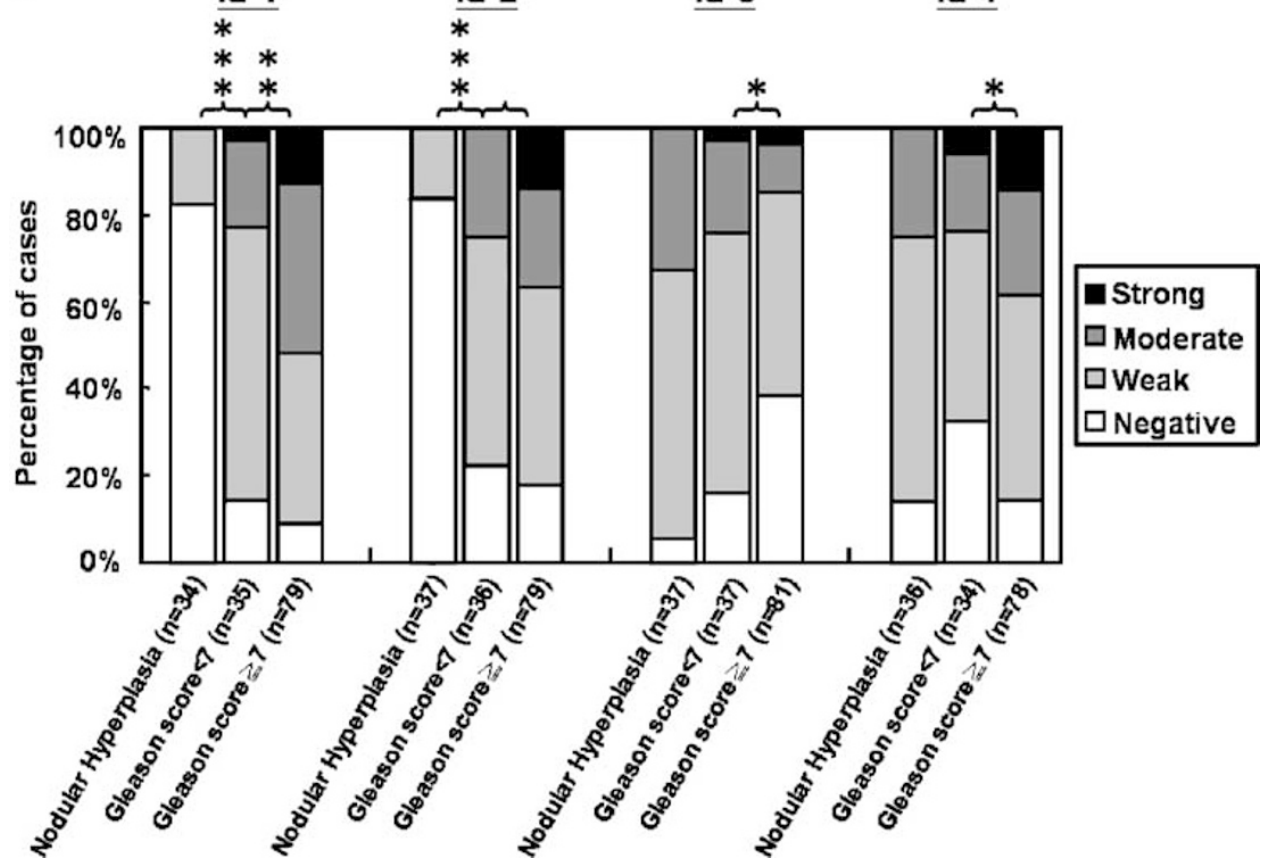

Figure 3 Summary of Id proteins immunohistochemical staining results in prostate specimens of different histological groups. (a) Percentage of nuclei stained positive for Id-2. (b) Percentage of nuclei stained positive for Id-4. (c) Cytoplasmic staining intensity for the four Id proteins. ${ }^{*},{ }^{* *},{ }^{* *}$ indicates statistical significant difference at $P<0.05,<0.01$ and $<0.001$ respectively. Cytoplasmic staining of Id-1 and nuclear staining of Id-2 are progressively increased from nodular hyperplasia, low-grade to high-grade prostate cancers. Significant difference between low- and high-grade prostate cancer is observed for cytoplasmic Id-3 and -4 staining. Nuclear staining of Id-4 in low-grade prostate cancer is significantly lower when compared with both nodular hyperplasia and high-grade prostate cancer.

\section{Association of Expressions of Id Proteins with Metastatic Potential of Prostate Cancer}

Metastatic progression is a major cause of death in prostate cancer patients. ${ }^{28}$ Biomarkers that accurately predict metastatic potential of the primary prostate cancer will be useful in patient management. We investigated whether expressions of Id proteins in prostate cancer could serve the purpose of being prognostic biomarkers by comparing the expression levels of the four Id proteins in Gleason score matched primary prostate cancer specimens. The metastatic group $(n=37)$ included cases with metastasis detected within 6 months of procurement of tumor sample, and the nonmetastatic group $(n=34)$ that included cases with no distant metastasis detected for at least 12 months follow-up. As very few patients with low-grade prostate cancer had developed metastatic disease within 6 months of follow-up, no statistical analysis could be performed and the data are not presented.

The results are summarized in Figure 4 and Table 3. Cytoplasmic staining of Id-1, $-2,-3$ and -4 in the high-grade prostate cancer specimens was not significantly different between the metastatic and nonmetastatic groups $(P=0.659,0.459,0.989$ and 0.488 , respectively) (Figure 4c). The nuclear staining score of Id-2 in the nonmetastatic group was $5.33 \pm 1.29 \%$ and it was significantly less than the $11.72 \pm 2.26 \%$ of the metastatic group $(P=0.038)$ 
a

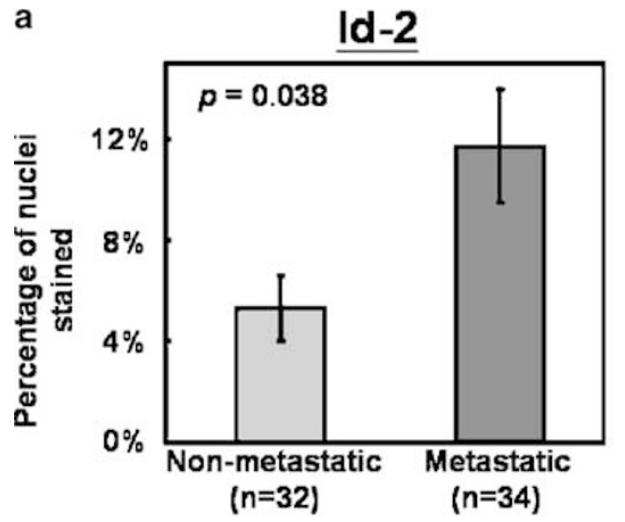

b

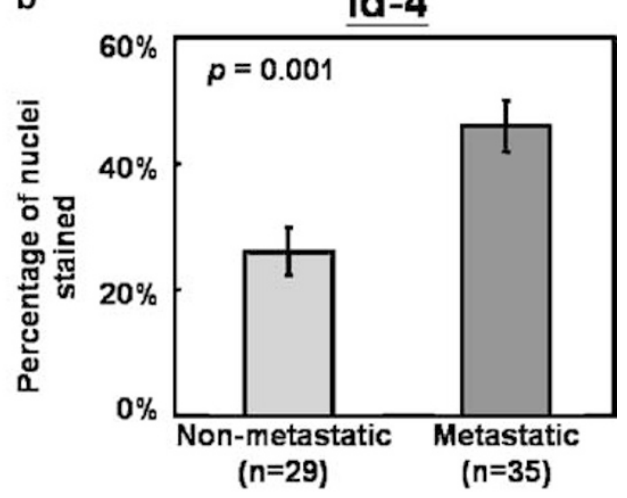

c

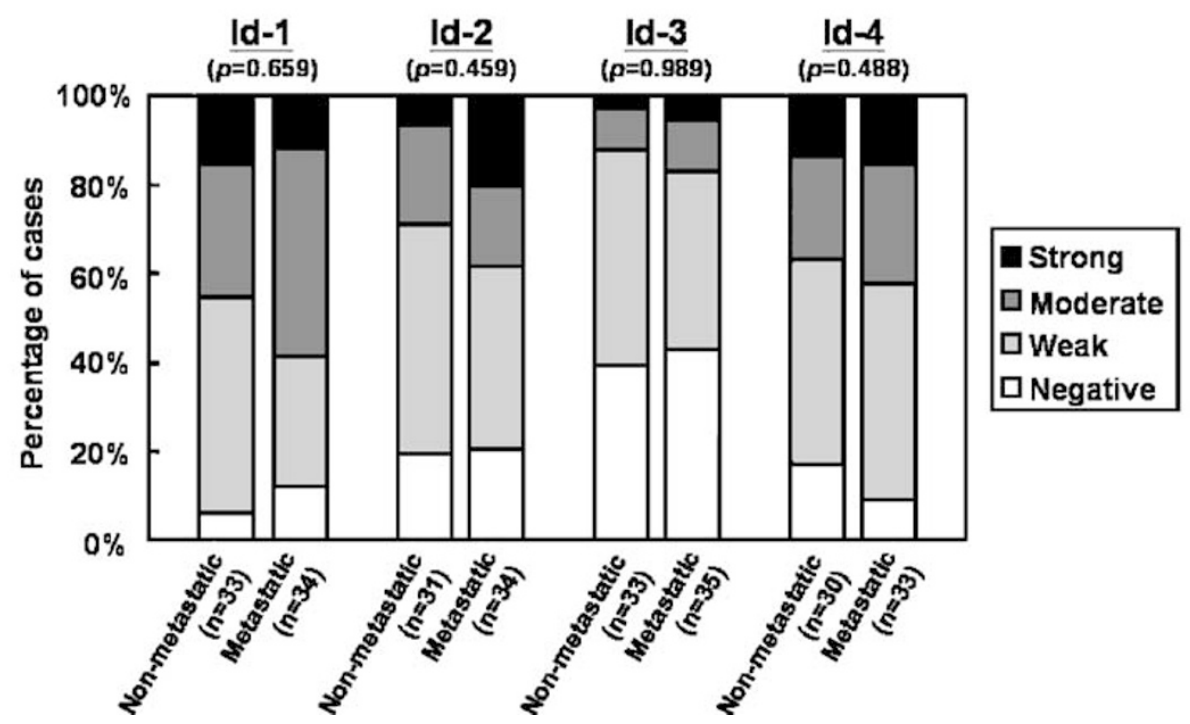

Figure 4 Summary of Id proteins immunohistochemical staining results in high-grade prostate cancers (Gleason score $\geq 7$ ) with differential metastatic status. (a) Percentage of nuclei stained positive for Id-2. (b) Percentage of nuclei stained positive for Id-4. (c) Cytoplasmic staining intensity for the four Id proteins. No significant difference in cytoplasmic staining was observed between the metastatic and nonmetastatic subgroups for the four Id proteins. Nuclear staining of Id-2 and -4 is significantly increased in the metastatic subgroup compared to the nonmetastatic subgroup. These results suggest that nuclear expression of Id-2 and -4 may play a positive role in promoting prostate cancer metastasis.

(Figure 4a). The nuclear staining score of Id-4 of the nonmetastatic group, which was $26.22 \pm 3.69 \%$, was also significantly lower than the $46.09 \pm 4.12 \%$ of the metastatic group $(P=0.001)$ (Figure $4 \mathrm{~b})$.

Since only Id-2 and -4 nuclear staining scores were significantly different between the two subgroups of high-grade prostate cancer, the risks of developing metastatic lesions with high nuclear expression of Id- 2 and -4 were estimated by odds ratio and $95 \%$ confidence interval through binary logistic regression analysis. The cutoff points for high-level expression for Id-2 and -4 were the median scores of these Gleason score matched subgroups, which are 1.47 and $32.4 \%$, respectively. More cases with high-level Id-2 nuclear staining scores $(61 \%, 20$ out of 33$)$ than those with low-level scores $(42 \%, 14$ out of 33$)$ developed metastatic lesion. However, high-level Id-2 nuclear staining did not significantly associate with a higher risk to develop distant metastatic lesions (odds ratio $=$ $2.088,95 \%$ confidence interval $=0.782-5.574$,
$P=0.142$ ). Metastasis was detected in 22 out of 32 (69\%) patients, whose prostate cancer showed highlevel Id-4 nuclear staining while only 13 out of 32 $(41 \%)$ patients with low-level staining developed metastatic lesion. The risk of patients with highlevel Id-4 nuclear staining in primary prostate cancer to develop metastatic lesion was significantly higher than those with low-level staining (odds ratio $=3.215,95 \%$ confidence interval $=1.150-$ 8.987, $P=0.026$ ). Based on these results, we suggest that nuclear expression of Id-4 may serve as a prognostic marker for predicting early metastatic disease in high-grade prostate cancer.

\section{Discussion}

We investigated, by immunohistochemistry, expressions of four members of the Id protein family in nodular hyperplasia $(n=40)$ and prostate cancer $(n=125)$. The staining results were correlated with 
clinico-pathological parameters. We observed dysregulation of the four Id proteins in prostate cancer, including upregulation of Id-1 and -2 and downregulation of Id-3 and -4 (Figures 1 and 2c) in prostate cancer specimens. Cytoplasmic staining of Id-1 and nuclear staining of Id-2 and -4 were positively correlated with Gleason score, suggesting their involvement in prostate cancer progression. Nuclear expression of Id-4 in primary prostate cancer specimens was associated with a higher risk to develop distant metastasis, suggesting that Id-4 might be involved in the metastatic process of prostate cancer. Taken together, our results suggest that Id proteins are differentially involved in the neoplastic transformation and cancer progression of prostate, and that Id-4 may be a potential marker for prediction of metastasis in high-grade prostate cancer. Our findings warrant further studies to investigate the exact roles played by the Id proteins in prostate cancer and the pathogenetic mechanisms involved.

It has been found that Id-1 negatively regulates differentiation of rat prostate epithelial cells in an androgen dependent fashion. ${ }^{29}$ Id-1 expression has previously been shown to be upregulated in prostate cancer and to correlate with Gleason grading. ${ }^{26}$ Our new results were similar. Participation of Id-1 in promoting metastasis varies among different types of cancer. ${ }^{12,30,31}$ Although invasiveness of prostate cancer cells could be increased by overexpressing Id-1 in a recent study, ${ }^{27}$ Id-1 expression was not significantly different between subgroups of highgrade prostate cancer of different metastatic outcome in the present study (Figure 4c). Two recent studies on breast cancer bone ${ }^{32}$ and lung ${ }^{33}$ metastasis showed that overexpression of Id-1 in breast cancer cells conferred higher metastatic ability to lung but not to bone. ${ }^{33}$ In our present study, 95\% patients of the metastatic group were diagnosed with bone metastasis, which may explain why Id-1 expression level is not significantly different between metastatic and nonmetastatic group.

Id-2 has been showed to directly bind to retinoblastoma protein and inhibits its tumor suppressor activity. ${ }^{34}$ In prostate cancer cell, forced expression of Id-2 increased their proliferation and invasion ability. ${ }^{27}$ Coppe et al have also reported that cytoplasmic Id-2 was upregulated in prostate cancer $(n=67)$ and positively correlated with Gleason grading, while nuclear staining was mainly found in high-grade prostate cancer. ${ }^{27}$ Our results of Id-2 staining showed a similar pattern. However, a correlation between cytoplasmic Id-2 staining and Gleason score could not be established (Figure 3c). Nuclear staining of Id-2 was mainly found in highgrade prostate cancer and significantly correlated with Gleason score (Figure 3a). The results suggest that while upregulation of Id-2 with its overexpression in the cytoplasm may be important in neoplastic transformation, nuclear expression of Id-2 is more likely to be related to cancer progression.
Despite an association of high-level nuclear expression of Id-2 with the development of distant metastasis within 6 months of follow-up (Figure 4a), the risk to develop metastasis with high Id-2 expression was not significantly increased.

Dysregulation of Id-3 has been described in several types of cancer including its upregulation in colorectal adenocarcinoma ${ }^{13}$ and pancreatic cancer, ${ }^{15}$ and its downregulation in ovarian cancer. ${ }^{19}$ In this study, Id-3 staining was not significantly different between nodular hyperplasia and lowgrade prostate cancer (Figure 3c). However, we observed a significant decrease in expression of Id-3 in high-grade prostate cancer as compared to low-grade prostate cancer (Figure 3c). A recent study on global gene methylation in prostate cancer cells has shown that Id-3 is methylated in PC3, an androgen-independent prostate cancer cell line. ${ }^{35}$ Evidence so far shows that downregulation of Id-3 may be involved in prostate cancer progression.

Hypermethylation and consequential reduced expression of Id-4 has been described in gastric cancer $^{17}$ and colorectal cancer. ${ }^{14}$ In the current study, nuclear staining score of Id-4 was significantly decreased in prostate cancer when compared to nodular hyperplasia (Figure 2b), suggesting that Id-4 may play a negative role in neoplastic transformation of prostate. On the other hand, nuclear staining of Id-4 was positively correlated with Gleason score (Figure 3b), suggesting that increased Id-4 expression may promote prostate cancer progression. Nuclear expression of Id-4 thus seemed to take different roles in prostate cancer initiation and progression. Others have found nuclear expression of Id-4 to be increased in the initiation and progression of breast cancer in rat. ${ }^{24}$ Id- 4 has been shown to positively correlate with invasion ability of a mouse breast cancer cell line in that same study. A significant association between Id-4 promoter hypermethylation and nodal metastasis in human breast cancer samples has been found..$^{25}$ Our study showed that high-level nuclear expression of Id-4 in high-grade primary prostate cancer was associated with higher risk to develop metastatic lesion (odds ratio $=3.215,95 \%$ confidence interval $=$ 1.150-8.987, $P=0.026$ ), suggesting that Id-4 may play a positive role in the metastatic process. Id-4 may be a potential marker for prediction of metastasis in prostate cancer patients.

Some mechanisms of how Id-1 and -2 interact have been proposed. Overexpression of Id-1 inhibits expression of p16, $,^{36,37} \mathrm{p} 21,{ }^{38}$ and $\mathrm{p} 27,{ }^{39}$ which leads to an increased activity of cyclin dependent kinase 2 (CDK2) and increased Retinoblastoma protein phosphorylation. Id-2, on the other hand, inhibits Retinoblastoma protein through direct binding and Id-2 itself is restrained by Retinoblastoma protein. ${ }^{34}$ Overexpression of Id-1 leads to increased Retinoblastoma protein phosphorylation and therefore increased liberation of Id-2 from Retinoblastoma protein and more free-Id2 is available for inhibition 
of $\mathrm{E}$ proteins to facilitate proliferation. ${ }^{40}$ In our study, we observed a positive correlation of cytoplasmic Id-1 and Id-2 expression $(r=0.288$, $P=0.003)$ in the prostate cancer specimens $(n=125)$. It is tempting to speculate that co-expression of Id-1 and Id-2 may cooperate to promote prostatic neoplastic transformation and cancer progression. Functional assay in cancer cells and mice models is warranted to test the hypothesis.

In summary, our study showed differential expressions of Id proteins in benign and malignant prostatic tissues. Evidence was provided that Id proteins might be differentially involved in neoplastic transformation, cancer progression and development of distant metastases. We demonstrated that nuclear expression of Id-4 could be a useful prognostic marker for high-grade prostate cancer with a high metastatic potential. Further investigations are needed to study the mechanisms involved, an understanding of which may provide the basis for a rational approach to the future development of targeted therapy against prostate cancer.

\section{References}

1 Konishi N, Shimada K, Ishida E, et al. Molecular pathology of prostate cancer. Pathol Int 2005;55: 531-539.

2 Linton KD, Hamdy FC. Early diagnosis and surgical management of prostate cancer. Cancer Treat Rev 2003;29:151-160.

3 Dale W, Bilir P, Han M, et al. The role of anxiety in prostate carcinoma: a structured review of the literature. Cancer 2005;104:467-478.

4 Oh WK, Kantoff PW. Management of hormone refractory prostate cancer: current standards and future prospects. J Urol 1998;160:1220-1229.

5 Zebedee Z, Hara E. Id proteins in cell cycle control and cellular senescence. Oncogene 2001;20:8317-8325.

6 Lasorella A, Uo T, Iavarone A. Id proteins at the crossroad of development and cancer. Oncogene 2001;20: 8326-8333.

7 Benezra R, Rafii S, Lyden D. The Id proteins and angiogenesis. Oncogene 2001;20:8334-8341.

8 Wong YC, Wang X, Ling MT. Id-1 expression and cell survival. Apoptosis 2004;9:279-289.

9 Fong S, Debs RJ, Desprez PY. Id genes and proteins as promising targets in cancer therapy. Trends Mol Med 2004;10:387-392.

10 Norton JD, Deed RW, Craggs G, et al. Id helix-loophelix proteins in cell growth and differentiation. Trends Cell Biol 1998;8:58-65.

11 Damdinsuren B, Nagano H, Kondo M, et al. Expression of Id proteins in human hepatocellular carcinoma: relevance to tumor dedifferentiation. Int J Oncol 2005; 26:319-327.

12 Schindl M, Oberhuber G, Obermair A, et al. Overexpression of Id-1 protein is a marker for unfavorable prognosis in early-stage cervical cancer. Cancer Res 2001;61:5703-5736.

13 Wilson JW, Deed RW, Inoue $\mathrm{T}$, et al. Expression of Id helix-loop-helix proteins in colorectal adeno- carcinoma correlates with p53 expression and mitotic index. Cancer Res 2001;61:8803-8810.

14 Umetani N, Takeuchi H, Fujimoto A, et al. Epigenetic inactivation of ID4 in colorectal carcinomas correlates with poor differentiation and unfavorable prognosis. Clin Cancer Res 2004;10:7475-7483.

15 Maruyama H, Kleeff J, Wildi S, et al. Id-1 and Id-2 are overexpressed in pancreatic cancer and in dysplastic lesions in chronic pancreatitis. Am J Pathol 1999;155: 815-822.

16 Jang TJ, Jung KH, Choi EA. Id-1 gene downregulation by sulindac sulfide and its upregulation during tumor development in gastric cancer. Int J Cancer 2006; 118:1356-1363.

17 Chan AS, Tsui WY, Chen X, et al. Downregulation of ID4 by promoter hypermethylation in gastric adenocarcinoma. Oncogene 2003;22:6946-6953.

18 Stighall M, Manetopoulos C, Axelson H, et al. High ID2 protein expression correlates with a favorable prognosis in patients with primary breast cancer and reduces cellular invasiveness of breast cancer cells. Int J Cancer 2005;115:403-411.

19 Arnold JM, Mok SC, Purdie D, et al. Decreased expression of the Id3 gene at 1 p36.1 in ovarian adenocarcinomas. Br J Cancer 2001;84:352-359.

20 Tsuchiya T, Okaji Y, Tsuno NH, et al. Targeting Id1 and Id3 inhibits peritoneal metastasis of gastric cancer. Cancer Sci 2005;96:784-790.

21 Lin CQ, Singh J, Murata K, et al. A role for Id-1 in the aggressive phenotype and steroid hormone response of human breast cancer cells. Cancer Res 2000;60: 1332-1340.

22 Itahana Y, Singh J, Sumida T, et al. Role of Id-2 in the maintenance of a differentiated and noninvasive phenotype in breast cancer cells. Cancer Res 2003; 63:7098-7105.

23 Beger C, Pierce LN, Kruger M, et al. Identification of Id4 as a regulator of BRCA1 expression by using a ribozyme-library-based inverse genomics approach. Proc Natl Acad Sci USA 2001;98:130-135.

24 Shan L, Yu M, Qiu C, et al. Id4 regulates mammary epithelial cell growth and differentiation and is overexpressed in rat mammary gland carcinomas. Am J Pathol 2003;163:2495-2502.

25 Umetani N, Mori T, Koyanagi K, et al. Aberrant hypermethylation of ID4 gene promoter region increases risk of lymph node metastasis in $\mathrm{T} 1$ breast cancer. Oncogene 2005;24:4721-4727.

26 Ouyang XS, Wang X, Lee DT, et al. Over expression of ID-1 in prostate cancer. J Urol 2002;167:25982602.

27 Coppe JP, Itahana Y, Moore DH, et al. Id-1 and Id-2 proteins as molecular markers for human prostate cancer progression. Clin Cancer Res 2004;10:20442051.

28 Zhan P, Lee EC, Packman K, et al. Induction of invasive phenotype by Casodex in hormone-sensitive prostate cancer cells. J Steroid Biochem Mol Biol 2002;83: 101-111.

29 Asirvatham AJ, Schmidt M, Gao B, et al. Androgens regulate the immune/inflammatory response and cell survival pathways in rat ventral prostate epithelial cells. Endocrinology 2006;147:257-271.

30 Takai N, Miyazaki T, Fujisawa K, et al. Id1 expression is associated with histological grade and invasive behavior in endometrial carcinoma. Cancer Lett 2001;165:185-193. 
31 Kebebew E, Peng M, Treseler PA, et al. Id1 gene expression is up-regulated in hyperplastic and neoplastic thyroid tissue and regulates growth and differentiation in thyroid cancer cells. J Clin Endocrinol Metab 2004;89:6105-6111.

32 Kang Y, Siegel PM, Shu W, et al. A multigenic program mediating breast cancer metastasis to bone. Cancer Cell 2003;3:537-549.

33 Minn AJ, Gupta GP, Siegel PM, et al. Genes that mediate breast cancer metastasis to lung. Nature 2005; 436:518-524.

34 Lasorella A, Noseda M, Beyna M, et al. Id2 is a retinoblastoma protein target and mediates signalling by Myc oncoproteins. Nature 2000;407:592-598.

35 Lodygin D, Epanchintsev A, Menssen A, et al. Functional epigenomics identifies genes frequently silenced in prostate cancer. Cancer Res 2005;65: 4218-4227.
36 Ohtani N, Zebedee Z, Huot TJ, et al. Opposing effects of Ets and Id proteins on p16INK4a expression during cellular senescence. Nature 2001;409:1067-1070.

37 Ouyang XS, Wang X, Ling MT, et al. Id-1 stimulates serum independent prostate cancer cell proliferation through inactivation of p16(INK4a)/pRB pathway. Carcinogenesis 2002;23:721-725.

38 Prabhu S, Ignatova A, Park ST, et al. Regulation of the expression of cyclin-dependent kinase inhibitor p21 by E2A and Id proteins. Mol Cell Biol 1997;17:5888-5896.

39 Everly Jr DN, Mainou BA, Raab-Traub N. Induction of Id1 and Id3 by latent membrane protein 1 of EpsteinBarr virus and regulation of $\mathrm{p} 27 / \mathrm{Kip}$ and cyclindependent kinase 2 in rodent fibroblast transformation. J Virol 2004;78:13470-13478.

40 Perk J, Iavarone A, Benezra R. Id family of helix-loophelix proteins in cancer. Nat Rev Cancer 2005;5: 603-614. 